\title{
INTEGRATED CONTINUITY CONDITIONS AND DEGREE OF APPROXIMATION BY POLYNOMIALS OR BY BOUNDED ANALYTIC FUNCTIONS $\left({ }^{1}\right)$
}

\author{
BY \\ J. L. WALSH AND H. G. RUSSELL
}

Introduction. In the study of uniform approximation to a function of a complex variable by polynomials or by bounded analytic functions, Lipschitz conditions have proved extremely useful in relating degree of approximation to continuity properties of the functions approximated. Parts of this theory are analogous to the older study (S. Bernstein, D. Jackson, de la Vallée Poussin, Montel) of approximation to real periodic functions by trigonometric sums, where Lipschitz conditions have also proved useful. Hardy and Littlewood $[2$, p. 633$]\left({ }^{2}\right)$ first pointed out that trigonometric approximation in the mean is likewise closely related to integrated Lipschitz conditions; proofs of the theorems stated by Hardy and Littlewood were first published by Quade [1]. The object of the present note is to indicate rapidly and with a minimum of detail, that degree of approximation in the mean by polynomials in the complex variable and by bounded analytic functions is also conveniently investigated by use of integrated Lipschitz conditions. The investigation leads naturally to the use also of classes of analytic functions satisfying integrated $Z$ ygmund and integral asymptotic conditions.

We shall approximate functions on an analytic Jordan curve or set of curves in the $z$-plane. Throughout the paper $\gamma$ denotes the unit circle, $C$ a single analytic Jordan curve or a finite number $\lambda$ of mutually exterior analytic Jordan curves $C_{j}, j=1,2, \cdots, \lambda$; if $\lambda=1, C_{1}=C$. A function $z=\chi_{j}(w)$, $w=r e^{i \theta}$, maps the closed interior of $\gamma:|w|=1$ onto the closed interior of $C_{j}$ one-to-one and conformally; $w=\Omega_{j}(z)$ denotes the function inverse to $z$ $=\chi_{j}(w) ; \bar{C}_{j}$ denotes the interior of $C_{j}$ together with $C_{j}$. Similarly, $w=\phi(z)$ maps the exterior of $C$ conformally (not necessarily one-to-one) onto $|w|>1$ so that $\infty=\phi(\infty) ; C_{R}:|\phi(z)|=R(>1)$ is the image in the exterior of $C$ of $|w|=R$ under this mapping. In this paper, the letter $s$ will denote arc-length measured on $C$, the letter $p$ a number not less than unity, the letter $k$ a non-negative integer unless otherwise indicated, the letters $M$ and $L$ with or without subscript constants independent of $n, z$ and $w$.

$A$ function $f(z)$ belongs to the class $H_{p}$ on $\gamma$ if it is analytic interior to $\gamma$ and if $M_{p}\left[f\left(r e^{i \theta}\right)\right]=\left\{\int_{0}^{2 n}\left|f\left(r e^{i \theta}\right)\right|^{p} d \theta\right\}^{1 / p}$ is bounded for $0<r<1$. A function $f(z)$

Presented to the Society, April 19, 1958; received by the editors March 7, 1958.

(1) This research was sponsored (in part) by the United States Air Force, Office of Scientific Research of the Air Research and Development Command.

(2) References in brackets refer to the bibliography at the end of the paper. 
belongs to the class $H_{p}$ on $C$ if $f\left[\chi_{j}(w)\right], j=1,2, \cdots, \lambda$, belongs to the class $H_{p}$ on $\gamma$. Smirnoff $[1$, p. 341] (see also Rudin [1, pp. 46-47]) has shown that the class $H_{p}$ on $\gamma$ is invariant under a one-to-one conformal mapping of the interior of $\gamma$ onto itself. If $f(z)$ belongs to $H_{p}$ on $\gamma$ [or $C$ ] boundary values of $f(z)$ for approach "in angle" exist almost everywhere on $\gamma$ [or $C]$ and $f(z)$ is of class $L^{p}$ there.

We shall approximate functions whose $k$ th derivatives belong to the class $H_{p}$ on $C$; these derivatives are also to have certain integral moduli of continuity $\omega_{p}(\delta)$ or generalized integral moduli of continuity $\omega_{p}^{*}(\delta)$ on $C$. If $f^{(k)}(z)$ is a function of class $H_{p}$ on $C$, and $f^{(k)}(z) \equiv F_{j}(s)$, where $s$ denotes arclength measured on $C_{j}$, then we set

$$
\begin{aligned}
\omega_{p j}\left(\delta ; f^{(k)}\right) & =\omega_{p j}\left(\delta ; F_{j}\right)=\omega_{p j}(\delta)=\max _{0<|h| \leqq \delta}\left\{\int_{C_{j}}\left|F_{j}(s+h)-F_{j}(s)\right| p d s\right\}^{1 / p}, \\
\omega_{p j}^{*}\left(\delta ; f^{(k)}\right) & =\omega_{p j}^{*}\left(\delta ; F_{j}\right)=\omega_{p j}^{*}(\delta) \\
& =\max _{0<|h| \leqq \delta}\left\{\int_{C_{j}}\left|F_{j}(s+h)+F_{j}(s-h)-2 F_{j}(s)\right|^{p} d s\right\}^{1 / p},
\end{aligned}
$$

respectively the integral modulus of continuity and the generalized integral modulus of continuity of $f^{(k)}(z)$ on $C_{j}$. The integral modulus of continuity $\omega_{p}\left(\delta ; f^{(k)}\right)=\omega_{p}(\delta)$ and the generalized integral modulus of continuity $\omega_{p}^{*}\left(\delta ; f^{(k)}\right)$ $=\omega_{p}^{*}(\delta)$ of $f^{(k)}(z)$ on $C$ are then defined to be respectively the maximum of $\omega_{p j}(\delta)$ and $\omega_{p j}^{*}(\delta), 1 \leqq j \leqq \lambda$. If $\omega_{p}(\delta) \leqq M \delta^{\alpha}, 0<\alpha<1$, or $\omega_{p}^{*}(\delta) \leqq M \delta$, then $f^{(k)}(z)$ satisfies respectively an integrated Lipschitz condition or an integrated Zygmund condition on $C$. These integral moduli have significance even if the function $f\left(e^{i \theta}\right)$ is defined only for the real variable $\theta$. In this case $f\left(e^{i \theta}\right) \equiv F(\theta)$ is said to belong to the Hardy and Littlewood class Lip $(\alpha, p)$ or the Zygmund class $\Lambda_{p}$.

We shall deal chiefly with functions which belong to the classes $H(k, \alpha, p)$, $0<\alpha<1$, or $Z(k, p)$ on an analytic Jordan curve $C$. The classes are defined as follows. Let $f(z)$ be analytic interior to $C$ and let $f^{(k)}(z)$ be of class $H_{p}$ on $C$. If $\omega_{p}\left(\delta ; f^{(k)}\right) \leqq M \delta^{\alpha}, 0<\alpha<1$, then $f(z)$ belongs to the class $H(k, \alpha, p)$ on $C$. If $\omega_{p}^{*}\left(\delta ; f^{(k)}\right) \leqq M \delta$, then $f(z)$ belongs to the class $Z(k, p)$ on $C$. If $k=0$, we write $f(z)=f^{(0)}(z)$. If $f(z)$ belongs to the class $H(k, \alpha, p)$ or $Z(k, p)$ on $C$ and $k \geqq 1$, then $f^{\prime}(z)$ belongs respectively to $H(k-1, \alpha, p)$ or $Z(k-1, p)$ on $C$; if $k \geqq 0$, the indefinite integral of $f(z)$ belongs (Theorem $C$ below) respectively to $H(k+1, \alpha, p)$ or $Z(k+1, p)$ on $C$.

If $p=\infty$, the function $f(z)$ is said to belong to the class $H(k, \alpha, \infty)$ or $Z(k, \infty)$ if $f(z)$ is analyiic interior to $C$ and continuous on $\bar{C}$, if $f^{(k)}(z) \equiv F(s)$ exists on $C$ in the one-dimensional sense, and if $F(s)$ satisfies the respective conditions $|F(s+h)-F(s)| \leqq M|h|^{\alpha}, 0<\alpha<1$, or $|F(s+h)+F(s-h)-2 F(s)| \leqq M|h|$. If $k=0$, the classes $H(0, \alpha, \infty)$ and $Z(0, \infty)$ are denoted by $H(\alpha)$ and $Z$ respectively. If $k=0$, and $f\left(e^{i \theta}\right)$ is a continuous function of the real variable 
$\theta$, the function $f\left(e^{i \theta}\right) \equiv F(\theta)$ belongs to the Hardy and Littlewood class Lip $\alpha$ or the Zygmund class $\Lambda$ if $F(\theta)$ satisfies the respective conditions $\mid F(\theta+h)$ $-F(\theta)|\leqq M| h \mid{ }^{\alpha}, 0<\alpha<1$, or $|F(\theta+h)+F(\theta-h)-2 F(\theta)| \leqq M|h|$.

If $C$ consists of a finite number of mutually exterior analytic Jordan curves, a function $f(z)$ belongs to the class $H(k, \alpha, p), 0<\alpha<1$, or $Z(k, p)$ on $C$ if $f(z)$ belongs to the respective class $H(k, \alpha, p)$ or $Z(k, p)$ on each component of $C$. An analogous definition holds for the classes $H(k, \alpha, \infty)$ and $Z(k, \infty)$.

For convenient reference we state as Lemma 1 certain results established by Walsh [1, pp. 92-93], Walsh and Sewell [1, Lemma 12.1], Sewell [1, Theorem 2.21, Lemma 8.1.7] and Western [1, Theorem 2], and as Theorem A results of Hardy and Littlewood established by Quade [1, Theorems 1-4] and results due to Zygmund [1, Theorem 9'].

Lemma 1. If $C$ is an analytic Jordan curve and $P_{n}(z)$ is a polynomial in $z$ of degree $n$ for which $\int_{C}\left|P_{n}(z)\right| p|d z| \leqq L^{p}$, then

$$
\begin{array}{rlrl}
\left|P_{n}(z)\right| & \leqq L^{\prime} L R^{n}, & & \text { zon } C_{R} ; \\
\left|P_{n}(z)\right| & \leqq L^{\prime} L n^{1 / p}, & & \text { zon } \bar{C} ; \\
\left\{\int_{C}\left|P_{n}^{\prime}(z)\right| p|d z|\right\}^{1 / p} & =L^{\prime} L n ; &
\end{array}
$$

in these inequalities $L^{\prime}$ is independent of $n$ and $z$ but depends on $p$ and $C$; in inequality (1.1), $L^{\prime}$ also depends on $C_{R}$. Inequality (1.1) is valid if $C$ is a finite number of mutually exterior analytic Jordan curves.

If $C$ is an analytic Jordan curve containing the origin in its interior and if $P_{n}(z, 1 / z)$ is a polynomial in $z$ and $1 / z$ of degree $n$ for which $\int_{C}\left|P_{n}(z, 1 / z)\right| p|d z|$ $\leqq L^{p}$, then

$$
\left|P_{n}(z, 1 / z)\right| \leqq L^{\prime} L R^{n}
$$

zon $\bar{A}$,

where $A$ is the region bounded by $|\phi(z)|=R$ and $|\Omega(z)|=1 / R ;$ in this inequality $L^{\prime}$ is independent of $n$ and $z$ but depends on $p, C$ and $R$.

Theorem A. A necessary and sufficient condition that a function $F(\theta)$ periodic of period $2 \pi$ can be uniformly approximated in the mean of order $p$ by trigonometric polynomials $T_{n}(\theta)$ of respective orders $n$ with the degree of approximation $1 / n^{k+\alpha}, 0<\alpha \leqq 1$, is that $F^{(k)}(\theta)$ belong to $\operatorname{Lip}(\alpha, p)$ if $0<\alpha<1$ and to $\Lambda_{p}$ if $\alpha=1$.

Preliminary results. The main conclusions of the paper require several preliminary theorems in addition to those already stated.

LEMma 2. Let the function $w=f(z)$ analytic on $\gamma:|z|=1$ transform $\gamma$ onto $\gamma^{\prime}:|w|=1$ one-to-one and so that $f^{\prime}(z) \neq 0$ on $\gamma$. Then an annulus $A$ containing $\gamma$ is transformed conformally and one-to-one onto an annulus $A^{\prime}$ containing $\gamma^{\prime}$. 
If the conclusion is false, for $n$ sufficiently large the annulus $A_{n}: 1-1 / n$ $<|z|<1+1 / n$ within which $f(z)$ is analytic contains two distinct points $\alpha_{n}$ and $\beta_{n}$ whose images are identical: $f\left(\alpha_{n}\right)=f\left(\beta_{n}\right)$. A subsequence $\alpha_{n_{k}}$ of the points $\alpha_{n}$ approaches a limt $\alpha_{0}$, necessarily on $\gamma$, and a subsequence of the points $\beta_{n_{k}}$ approaches a limit $\beta_{0}$, necessarily on $\gamma$. Then if $\alpha_{0} \neq \beta_{0}$ we have $f\left(\alpha_{n_{k}}\right)=f\left(\beta_{n_{k}}\right), f\left(\alpha_{0}\right)=f\left(\beta_{0}\right)$; if $\alpha_{0}=\beta_{0}$ we have $f^{\prime}\left(\alpha_{0}\right)=0$; in any case we have reached a conclusion contrary to the hypothesis on $f(z)$.

TheOREM B. Let the function $\phi(\theta)$ have period $2 \pi$ and let $\phi^{(k)}(\theta)$ be of class $\operatorname{Lip}(\alpha, p)$ in $\theta$; let $\theta\left(\theta_{1}\right)$ be a transformation analytic for all $\theta$ and one-to-one, with $\theta_{1}^{\prime}(\theta) \neq 0$, and $\theta\left(\theta_{1}+2 \pi\right) \equiv \theta\left(\theta_{1}\right)+2 \pi$. Then $d^{k} \phi\left[\theta\left(\theta_{1}\right)\right] / d \theta_{1}^{k}$ is of class Lip $(\alpha, p)$ in $\theta_{1}$.

We interpret the range $-\infty<\theta<+\infty$ on $\gamma: z=e^{i \theta}$. From the hypothesis on $\phi(\theta)$ it follows that there exist trigonometric polynomials of respective degrees $n$, namely polynomials $p_{n}(z, 1 / z)$ of respective degrees $n$ in $z$ and $1 / z$, such that

$$
\int_{\gamma}\left|\phi(-i \log z)-p_{n}(z, 1 / z)\right| p|d z| \leqq L / n^{(k+\alpha) p} .
$$

The functions $p_{n}(z, 1 / z)$ then satisfy the inequality

$$
\int_{\gamma}\left|p_{n}(z, 1 / z)\right|^{p}|d z| \leqq L_{1}
$$

and by Lemma 1 , the inequality

$$
\left|p_{n}(z, 1 / z)\right| \leqq L_{2} R^{n}, \quad z \text { in } A,
$$

where $A$ is the annulus whose existence is asserted in Lemma 2, for the transformation of $\gamma$ onto $\gamma^{\prime}: w=e^{i \theta_{1}(\theta)}$. Denote by $A^{\prime}$ the image of $A$ under this transformation. Then in the $w$-plane we may express (1) as

$$
\int_{\gamma^{\prime}}\left|\phi\left[\theta\left(\theta_{1}\right)\right]-p_{n}(z, 1 / z)\right| p\left|d \theta / d \theta_{1}\right| d \theta_{1} \leqq L / n^{(k+\alpha) p},
$$

which by the boundedness of $1 /\left|d \theta / d \theta_{1}\right|$ implies

$$
\int_{\gamma^{\prime}}\left|\phi\left[\theta\left(\theta_{1}\right)\right]-f_{n}(w)\right|^{p} d \theta_{1} \leqq L_{3} / n^{(k+\alpha) p}, \quad f_{n}(w) \equiv p_{n}(z, 1 / z)
$$

and we have

$$
\left|f_{n}(w)\right| \leqq L_{2} R^{n}
$$
$z$ in $A^{\prime}$.

The conclusion now follows as in Theorem 8 below.

This method shows simultaneously that if $\phi^{(k)}(\theta)$ is of class $\Lambda_{p}$ in $\theta$, so also is $d^{k} \phi\left[\theta\left(\theta_{1}\right)\right] / d \theta_{1}^{k}$ in $\theta_{1}$. 
REMARK. Theorem $B$ remains essentially valid if $\phi(\theta)$ is periodic with period $\tau \neq 2 \pi$ and $\theta\left(\theta_{1}\right)$ has corresponding properties.

Theorem C. The indefinite integral $F(z)$ of a function of class $H_{p}$ on an analytic Jordan curve $C$ is continuous on $\bar{C}$ and satisfies the condition $\omega_{p}(\delta ; F)$ $\leqq M \delta$ on $C$.

Let $f(z)$ be a function of class $H_{p}$ on $C$. Then $h(w) \equiv f[\chi(w)]$ belongs to the class $H_{p}$ on $\gamma$, and, since $\left|\chi^{\prime}(w)\right| \leqq L$ if $|w| \leqq 1$, so does the function $g(w)$ $\equiv h(w) \cdot \chi^{\prime}(w)$. F. Riesz $[1$, p. 95] has proved that the indefinite integral of a function of class $H_{1}$ on $\gamma$ is continuous on $\bar{\gamma}$. By Riesz's theorem, the function $G(w) \equiv \int_{0}^{w} g(w) d w$ is continuous on $\bar{\gamma}$, where the path of integration between 0 and $w$ lies interior to $\gamma$. Since $G(w) \equiv G[\Omega(z)] \equiv F(z) \equiv \int_{z_{0}}^{z} f(z) d z$, and $\Omega(z)$ is analytic on $\bar{C}$, the function $F(z)$ is analytic interior to $C$ and continuous on $\bar{C}$.

The fact that $\omega_{p}(\delta ; G) \leqq M \delta$ on $\gamma$ follows by a lemma of Hardy and Littlewood [2, p. 619, Lemma 10], namely that "if $f(\theta)$ is the $\alpha$ th integral of a function of $L^{p}$, then $f(\theta)$ belongs to $\operatorname{Lip}(\alpha, p), " 0<\alpha \leqq 1, p \geqq 1$. In considering the function $G(w) \equiv \int_{0}^{w} g(w) d w$, the path for $|w|=1$ may be taken along a radius, or may be taken in part along an arc of $\gamma$, by the boundedness of $\int_{0}^{2 \pi}\left|g\left(r e^{i \theta}\right)\right| d \theta$ for $0<r \leqq 1$. Thus $F(z) \equiv \int_{z_{0}}^{z} f(z) d z$ may be taken along $C$. By the remark following Theorem B the function $F(z)$ satisfies the condition $\omega_{p}(\delta ; F)$ $\leqq M \delta$ on $C$.

Lemma 3. If $g(z)$ is analytic in $\bar{C}$, and if $f(z)$ is of class $H(0, \alpha, p)$ or $Z(0$, $p)$ on $C$, then $f(z) g(z)$ is also of class $H(0, \alpha, p)$ or $Z(0, p)$ on $C$.

The proof follows from simple algebraic equations and the use of Minkowski's inequality for integrals.

Lemma 4. If $f(z)$ belongs to $H(k, \alpha, p)$ or to $Z(k, p)$ on an analytic Jordan curve $C$, then $f[\chi(w)]=F_{1}(w)$ belongs to the corresponding class on $\gamma ;$ if $g(w)$ belongs to $H(k, \alpha, p)$ or to $Z(k, p)$ on $\gamma$, then $g[\Omega(z)]=h(z)$ belongs to the corresponding class on $C$.

If $k=0$, the conclusion of the lemma follows from Theorem B and the invariance of class $H_{p}$ under conformal mapping. If $k \geqq 1$, the function $f^{(k-1)}(z)$ satisfies the condition $\omega_{p}\left(\delta ; f^{(k-1)} \leqq M \delta\right.$ on $C$, by Theorem $\mathrm{C}$; if $k \geqq 2$, the functions $f^{(j)}(z), j \leqq k-2$, have continuous derivatives. By the proof for $k=0$, the function $f^{(k)}[\chi(w)]$ belongs to $H(0, \alpha, p)$ or $Z(0, p)$ on $\gamma$ when $f^{(k)}(z)$ belongs to the corresponding class on $C$; by the proof of Theorem $\mathrm{C}$, the functions $f^{(j)}[\chi(w)], j \leqq k-1$, satisfy the condition $\omega_{p}\left(\delta ; f^{(j)}[\chi(w)]\right)$ $\leqq M \delta$ on $\gamma$; by hypothesis $\chi(w)$ is analytic on $\gamma$. From the equations $F_{1}(w)$ $=f^{\prime}(z) \chi^{\prime}(w), F_{1}^{\prime \prime}(w)=f^{\prime \prime}(z)\left[\chi^{\prime}(w)\right]^{2}+f^{\prime}(z) \chi^{\prime \prime}(w), \cdots$, and Lemma 3 , the first half of the lemma follows. The second half is proved similarly.

TheOREM D. If $f(z)$ belongs to $H(k, \alpha, p)[0<\alpha<1]$ or to $Z(k, p)[\alpha=1]$ on $\gamma$, there exist polynomials $\pi_{n}(z)$ in $z$ of degree $n$ such that 


$$
\int_{\gamma}\left|f(z)-\pi_{n}(z)\right| p|d z| \leqq \frac{M}{n^{(k+\alpha) p}} .
$$

By the method of proof of Lemma 4, from the equation $\partial^{k} f(z) / \partial \theta^{k}$ $\equiv \sum_{j=1}^{\boldsymbol{k}} c_{j} z^{i} f^{(j)}(z),|z|=1, c_{j}$ constants, it follows that the function $f\left(e^{i \theta}\right)$ $=F(\theta)$ belongs to $H(k, \alpha, p)$ or $Z(k, p)$ on $\gamma$. By Theorem A, trigonometric polynomials $t_{n}(\theta)$ of order $n$ exist such that

$$
\int_{0}^{2 \pi}\left|F(\theta)-t_{n}(\theta)\right|^{p} d \theta \leqq \frac{M}{n^{(k+\alpha) p}} .
$$

Quade [1, Theorem 3] follows de la Vallée Poussin, who has shown $[1, \S 31]$ the polynomials $t_{n}(\theta)$ to exist in the form

$$
t_{n}(\theta)=\frac{a_{0}}{2}+\sum_{\nu=1}^{n} d_{n, \nu}\left(a_{\nu} \cos \nu \theta+b_{\nu} \sin \nu \theta\right),
$$

where $a_{\nu}$ and $b_{\nu}$ are the Fourier coefflcients of $F(\theta)$ and $d_{n, \nu}$ are independent of $F(\theta)$ but depend on $k$, that is, the polynomials $t_{n}(\theta)$ are formed by applying a particular method of summation to the Fourier sums for $F(\theta)$. For $z=e^{i \theta}$, we have $t_{n}(\theta)=\sum_{\nu=0}^{n} d_{n, \nu} c_{\nu} z^{\nu}$, where $2 c_{\nu}=a_{\nu}-i b_{\nu}$, hence $t_{n}(\theta)$ is a polynomial $\pi_{n}(z)$ in $z$ of degree $n$ (i.e. of power series type) for which (6) holds.

Problem $\alpha$. We denote by Problem $\alpha$ the study of the relation between continuity properties on $C$ and degree of approximation on $C$, whereas we denote by Problem $\beta$ the study of the relation between continuity properties on $C_{\rho}$ (interior to which $f(z)$ is supposed analytic) and degree of approximation to $f(z)$ on $C$. Our main results follow.

THEOREM 1. Let $C$ consist of a finite number $\lambda$ of mutually exterior analytic Jordan curves $C_{j}$. If $f(z)$ belongs to $H(k, \alpha, p)[0<\alpha<1]$ or to $Z(k, p)[\alpha=1]$ on $C$, there exist polynomials $\pi_{n}(z)$ in $z$ of respective degrees $n$ such that

$$
\int_{C}\left|f(z)-\pi_{n}(z)\right|^{p}|d z| \leqq \frac{M}{n^{(k+\alpha) p}}, \quad 0<\alpha \leqq 1 .
$$

By Lemma 4 , the functions $f\left[\chi_{j}(w)\right]$ belong to $H(k, \alpha, p)$ or to $Z(k, p)$ on $\gamma$ when $f(z)$ belongs to the corresponding class on $C$. By Theorem $\mathrm{D}$ there exist polynomials $\pi_{j, n}(w)$ in $w$ of degree $n$ such that

$$
\int_{0}^{2 \pi}\left|f\left[\chi_{j}(w)\right]-\pi_{j, n}(w)\right| p d \theta \leqq \frac{M_{1}}{n^{(k+\alpha) p}}, \quad|w|=1 .
$$

Let $\pi_{j, n}(w) \equiv F_{j, n}(z)$. Since $\chi_{j}^{\prime}(w) \neq 0$ on $C_{j}$, we have

$$
\int_{C}\left|f(z)-F_{j, n}(z)\right|^{p}|d z| \leqq \frac{M_{2}}{n^{(k+\alpha) p}} .
$$


For some number $R>1$ the curves $C_{j}^{\prime}:\left|\Omega_{j}(z)\right|=R$ are analytic and mutually exterior and each $\Omega_{j}(z)$ is analytic in the closed interior of the corresponding $C_{j}^{\prime}$. From (7), the hypothesis on $f(z)$, and Lemma 1 , we have

$$
\left|\pi_{j, n}(w)\right| \leqq L^{\prime} R^{n}, w \text { on } \gamma_{R}, L^{\prime} \text { independent of } \pi_{j, n} \text { and } n,
$$

and hence

$$
\left|F_{j, n}(z)\right| \leqq L^{\prime} R^{n}, z \text { on } C_{j}^{\prime} .
$$

Using the method of Curtiss [1, pp. 875-877] we approximate to $F_{n}(z)$ $\equiv F_{j, n}(z)$ by polynomials $P_{q n}(z)$ of respective degrees $q n$ which interpolate to $F_{n}(z)$ in $q n+1$ equally distributed points on $C$ and are such that

$$
\left|F_{n}(z)-P_{q n}(z)\right| \leqq M_{3} \eta^{n}, \quad \eta<1, z \text { on } C \text {; }
$$

thus by (7)

$$
\int_{C}\left|f(z)-P_{q n}(z)\right|^{p}|d z| \leqq \frac{M_{4}}{n^{(k+\alpha) p}} ;
$$

polynomials $\pi_{n}(z)$ defined by $\pi_{n}(z) \equiv 0, n<q, \pi_{n}(z) \equiv P_{q j}(z), q j \leqq n<q(j+1)$, $j=1,2, \cdots$, then yield the theorem.

Obviously the conclusion of Theorem 1 holds if the $\pi_{n}(z)$ are extremal polynomials of respective degrees $n$ approximating to $f(z)$ on $C$ in the sense of least pth powers.

Theorem 2. Let $C$ be an analytic Jordan curve. Let $F(z)$ and polynomials $\pi_{n}(z)$ in $z$ of respective degrees $n$ exist such that

$$
\int_{C}\left|F(z)-\pi_{n}(z)\right| p|d z| \leqq \frac{M}{n^{(k+\alpha) p}}, \quad 0<\alpha \leqq 1 .
$$

Then $F(z)$ is equivalent (i.e. equal a.e.) on $C$ to a function $f(z)$ of class $H(k, \alpha, p)$ on $C$ if $0<\alpha<1$ and of class $Z(k, p)$ if $\alpha=1$. If $\alpha p>1$, the function $f^{(k)}(z)$ belongs to $H(\alpha-1 / p), 0<\alpha \leqq 1$, on $\bar{C}$, and we have

$$
\left|f(z)-\pi_{n}(z)\right| \leqq \frac{M_{1}}{n^{k+\alpha-1 / p}}, \quad \text { zon } \bar{C} .
$$

We adapt the method of proof used by de la Vallée Poussin [1, Chapter III] for $p=\infty$ and $f(\theta)$ a periodic function of period $2 \pi$.

From (8) we obtain

$$
\int_{C}\left|\pi_{2^{j+1}}(z)-\pi_{2^{j}}(z)\right| p|d z| \leqq \frac{M^{2}}{\left(2^{j}\right)^{(k+\alpha) p}} .
$$
have

Let $q_{j}(z) \equiv \pi_{2}{ }^{i+1}(z)-\pi_{2}{ }^{j}(z)$. From (10) and repeated use of Lemma 1, we 


$$
\int_{C}\left|q_{j}^{(\mu)}(z)\right|^{p}|d z| \leqq \frac{M_{3}}{\left(2^{j}\right)^{(k+\alpha-\mu) p}},
$$

and from (11) and Lemma 1,

$$
\left|q_{j}^{(\mu)}(z)\right| \leqq \frac{M_{4}}{\left(2^{j}\right)^{k+\alpha-\mu-1 / p}},
$$

Let $k=0$. For $z$ interior to $C$ and $|t-z| \geqq d>0$, inequality (8) yields

$$
\left|q_{j}(z)\right| \leqq \frac{1}{2 \pi} \int_{C} \frac{\left|q_{j}(t)\right|}{|t-z|}|d t| \leqq \frac{M_{5}}{2^{j} d},
$$

and by (13) and (10), the sequence $\left\{\pi_{2}{ }^{i}(z)\right\}$ converges uniformly on any closed set interior to $C$ to an analytic function $f(z)$ and in the mean on $C$ to a function of class $L^{p}$. We now prove that $f(z)$ belongs to the class $H_{p}$ on $C$. Let $F(w), \Pi_{2^{m}}(w)$ and $Q_{j}(w)$ denote respectively $f[\chi(w)], \pi_{2^{m}}[\chi(w)]$ and $q_{j}[\chi(w)]$, and let $w=r e^{i \theta}$. On any closed set interior to $\gamma$ we have

$$
F(w)-\Pi_{2^{m}}(w)=\sum_{j=m}^{\infty} Q_{j}(w),
$$

so that

$$
M_{p}\left[F\left(r e^{i \theta}\right)-\Pi_{2^{m}}\left(r e^{i \theta}\right)\right] \leqq M_{p}\left[\sum_{j=m}^{\infty} Q_{j}\left(r e^{i \theta}\right)\right] \leqq \sum_{j=m}^{\infty} M_{p}\left[Q_{j}\left(r e^{i \theta}\right)\right], r<1 .
$$

Since $\int_{C}\left|q_{j}(z)\right|^{p}|d z|=\int_{\gamma}\left|Q_{j}(w)\right|{ }^{p}\left|\chi^{\prime}(w)\right||d w|$ and since $\left|\chi^{\prime}(w)\right| \geqq L>0$ on $\gamma$, from inequality (10) we obtain

$$
M_{p}\left[Q_{j}\left(e^{i \theta}\right)\right]=\left\{\int_{\gamma}\left|Q_{j}(w)\right| p|d w|\right\}^{1 / p} \leqq \frac{M_{6}}{2^{j \alpha}} .
$$

The functions $\Pi_{2^{m}}(w)$ and $Q_{j}(w)$ analytic on $\gamma$ are of class $H^{p}$ on $\gamma$, so that with the use of (14) and (15) the inequality

$$
M_{p}\left[F\left(r e^{i \theta}\right)\right] \leqq M_{p}\left[\Pi_{2^{m}}\left(r e^{i \theta}\right)\right]+M_{p}\left[F\left(r e^{i \theta}\right)-\Pi_{2^{m}}\left(r e^{i \theta}\right)\right]
$$

yields

$$
\begin{aligned}
M_{p}\left[F\left(r e^{i \theta}\right)\right] & \leqq M_{7}+\sum_{j=m}^{\infty} M_{p}\left[Q_{j}\left(r e^{i \theta}\right)\right] \leqq M_{7}+\sum_{j=m}^{\infty} M_{p}\left[Q_{j}\left(e^{i \theta}\right)\right] \\
& \leqq M_{7}+\frac{M_{8}}{2^{m \alpha}}, \text { where } M_{7} \text { and } M_{8} \text { are independent }
\end{aligned}
$$

of $r$. Since $F(w)$ is analytic interior to $\gamma$ and $M_{p}\left[F\left(r e^{i \theta}\right)\right]$ is bounded for $0<r<1, F(w)$ is of class $H_{p}$ on $\gamma$, and $f(z)$ is of class $H_{p}$ on $C$. Thus $f(z)-\pi_{2^{m}}(z)$ is also of class $H_{p}$ on $C$ and 


$$
\int_{C}\left|f(z)-\pi_{2^{m}}(z)\right| p|d z| \leqq \frac{M_{9}}{2^{m \alpha p}} .
$$

The function $f(z)$ is equivalent on $C$ to $F(z)$ since

$$
\begin{aligned}
\int_{C} \mid F(z)- & \left.f(z)\right|^{p}|d z| \\
& \leqq 2^{p-1}\left[\int_{C}\left|F(z)-\pi_{2^{m}}(z)\right|^{p}|d z|+\int_{C}\left|f(z)-\pi_{2^{m}}(z)\right| p|d z|\right]
\end{aligned}
$$

compare (8).

Let $k=1$. Inequalities (12) and (13) imply that $\left\{\pi_{2}{ }^{i}(z)\right\}$ converges uniformly on $\bar{C}$ to a function $f(z)$ analytic interior to $C$, continuous on $\bar{C}$, equivalent to $F(z)$ on $C$, while $\left\{\pi_{2}^{\prime}(z)\right\}$ converges in the mean on $C$ to a function $g_{1}(z)$ of class $L^{p}$ on $C$. Since

$$
\begin{aligned}
\left|\int_{C}\left\{\sum_{j=m}^{n} q_{j}^{\prime}(t)-\left[g_{1}(t)-\pi_{2^{m}}^{\prime}(t)\right]\right\} d t\right| & \\
& \leqq M_{10}\left\{\int_{C}\left|\sum_{j=m}^{n} q_{j}^{\prime}(t)-\left[g_{1}(t)-\pi_{2^{m}}^{\prime}(t)\right]\right|^{p}|d t|\right\}^{1 / p}
\end{aligned}
$$

and the right member approaches zero with $1 / n$, we have

$$
\sum_{j=m}^{\infty} \int_{\zeta}^{z} q_{j}^{\prime}(t) d t=\int_{\zeta}^{z}\left[g_{1}(t)-\pi_{2^{m}}^{\prime}(t)\right] d t
$$

where $\zeta$ is a fixed point and $z$ a variable point on $C$. Integration gives

$$
f(z)-f(\zeta)=\int_{\zeta}^{z} g_{1}(t) d t
$$

and $g_{1}(z)=f^{\prime}(z)$ almost everywhere on $C$. By the argument for $k=0, f^{\prime}(z)$ is of class $H_{p}$ on $C$.

If $k>1$, inequalities (11) and (12) and repetition of the above arguments imply the existence of $f^{(k)}(z)$ of class $H_{p}$ on $C$.

If $k \geqq 0$, if $\alpha p>1$, inequality (8) implies [Sewell, 1 , Theorems 4.2 .1 and 4.2.2] that $f^{(k)}(z)$ is of class $H(\alpha-1 / p)$ on $\bar{C}$ and that (9) is valid.

We outline the proof that $f^{(k)}(z)$ belongs to $Z(0, p)$ if $\alpha=1$. The proof that $f^{(k)}(z)$ belongs to $H(0, \alpha, p)$ if $0<\alpha<1$ is similar.

Let $z=\tau(s)$ be the equation of $C$. Let $h$ be arbitrary but fixed, $0<h<1 / 2$, and let $N$ be a number such that $2^{N} \leqq h^{-1}<2^{N+1}$. Let $G(s)=\pi_{2}^{(k)}[\tau(s+h)]$ $+\pi_{2}^{(k)}[\tau(s-h)]-2 \pi_{2}^{(k)}[\tau(s)]$ and $G_{j}^{(k)}(s)=q_{j}^{(k)}[\tau(s+h)]+q_{j}^{(k)}[\tau(s-h)]$ $-2 q_{j}^{(k)}[\tau(s)], j \geqq 1$. We write 


$$
\begin{aligned}
&\left\{\int_{C}\left|f^{(k)}[\tau(s+h)]+f^{(k)}[\tau(s-h)]-2 f^{(k)}[\tau(s)]\right| p d s\right\}^{1 / p} \\
& \leqq\left\{\int_{C}|G(s)| p d s\right\}^{1 / p}+\left(\sum_{j=1}^{N}+\sum_{j=N+1}^{\infty}\right)\left\{\int_{C}\left|G_{j}^{(k)}(s)\right|^{p} d s\right\}^{1 / p}
\end{aligned}
$$

Each term on the right is not greater than $L|h|$. For the first term we have

$$
\begin{aligned}
& \left\{\int_{C}|G(s)| p d s\right\}^{1 / p} \leqq\left\{\int_{C} \mid \pi_{2}^{(k)}\left[\tau(s+h)-\left.\pi_{2}^{(k)}[\tau(s)]\right|^{p} d s\right\}^{1 / p}\right. \\
& +\left\{\int_{C}\left|\pi_{2}^{(k)}[\tau(s)]-\pi_{2}^{(k)}[\tau(s-h)]\right|^{p} d s\right\}^{1 / p} \\
& \leqq L|h| \text {; }
\end{aligned}
$$

for the third term,

$$
\begin{aligned}
\left\{\int_{C}\left|G_{j}^{(k)}(s)\right|^{p} d s\right\}^{1 / p} & \\
\leqq & \left\{\int_{C}\left|q_{j}^{(k)}[\tau(s+h)]\right|^{p} d s\right\}^{1 / p}+\left\{\int_{C}\left|q_{j}^{(k)}[\tau(s-h)]\right|^{p} d s\right\}^{1 / p} \\
& \quad+2\left\{\int_{C}\left|q_{j}^{(k)}[\tau(s)]\right|^{p} d s\right\}^{1 / p} \\
\leqq & L_{1} 2^{-i} ;
\end{aligned}
$$

for the second term, with the use of inequality (11) when $\mu=k+2$, we have

$$
\begin{aligned}
\left\{\int_{C}\left|G_{j}^{(k)}(s)\right|^{p} d s\right\}^{1 / p} & \leqq \int_{0}^{h}\left\{\int_{C}\left|q_{j}^{(k+1)}[\tau(\zeta+s)]-q_{j}^{(k+1)}[\tau(\zeta+s-h)]\right|^{p} d s\right\}^{1 / p} d \zeta \\
& \leqq L_{2} 2^{j} \int_{0}^{h}|\tau(\zeta+s)-\tau(\zeta+s-h)| d \zeta \\
& \leqq L_{3} 2^{i} h^{2} .
\end{aligned}
$$

Since $\sum_{j=N+1}^{\infty} 2^{-i} \leqq 2^{-N} \leqq 2|h|$ and $\sum_{j=1}^{N} 2^{i} h^{2} \leqq L_{4} 2^{N} h^{2} \leqq L_{5}|h|$, the conclusion follows.

Problem $\beta$. The definition of the classes $H(k, \alpha, p)$ and $Z(k, p)$ may be enlarged to include the case that $k$ is a negative integer. A function $f(z)$ is said to be of class $H(k, \alpha, p)$ or $Z(k, p)$ on an analytic Jordan curve $C$ when $k$ is a negative integer if $f[\chi(w)]$ is respectively of class $H(k, \alpha, p)$ or $Z(k, p)$ on $\gamma$. The function $f(z)=f\left(r e^{i \theta}\right)$ is said to be of class $H(k, \alpha, p)$ on $\gamma$ when $k$ is a negative integer if $f(z)$ is analytic interior to $\gamma$ and $M_{p}\left[f\left(r e^{i \theta}\right)\right] \leqq M(1-r)^{k+\alpha}$, 
$r<1$. This classification is based on results of Hardy and Littlewood [2, Theorem 3; 3, Theorem 46]: (a) A necessary and sufficient condition that a function $f(z)$ analytic interior to $\gamma$ belong to $H(0, \alpha, p)$ on $\gamma, 0<\alpha<1$, is that $M_{p}\left[f^{\prime}\left(r e^{i \theta}\right)\right] \leqq M(1-r)^{\alpha-1}, r<1$; (b) If $M_{p}\left[f\left(r e^{i \theta}\right)\right] \leqq M(1-r)^{\mu}, \mu<0$, then $M_{p}\left[f^{\prime}\left(r e^{i \theta}\right)\right] \leqq M_{1}(1-r)^{\mu-1} ; \quad$ (c) If $M_{p}\left[f\left(r e^{i \theta}\right)\right] \leqq M(1-r)^{\mu}, \quad \mu<-1$, then $M_{p}\left[F\left(r e^{i \theta}\right)\right] \leqq M_{2}(1-r)^{\mu+1}$, where $F(z)$ is any indefinite integral of $f(z)$. From these properties and the extended definition of $H(k, \alpha, p)$ it follows that on $\gamma$ and on $C$ for each $\alpha$ and $p$ we have a sequence of classes $\cdots, H(-2, \alpha, p)$, $H(-1, \alpha, p), H(0, \alpha, p), H(1, \alpha, p), H(2, \alpha, p), \cdots$; each function of a class is the derivative of a function of the next higher class and the indefinite integral of a function of the next lower class.

The invariance of the classes $H(k, \alpha, p)$ under conformal mapping of the interior of $\gamma$ onto itself is readily proved. Let $z=\chi(w)=\lambda(w-a) /(1-\bar{a} w)$, $|\lambda|=1,|a|<1$. If $k=-1$, by (a) the indefinite integral $F(z)$ belongs to $H(0, \alpha, p)$ on $\gamma$; this class is invariant under conformal mapping, so that $F[\chi(w)]$ belongs to $H(0, \alpha, p)$ on $\gamma$. Hence by $(\mathrm{a}), M_{p}\left[d F\left[\chi\left(r e^{i \theta}\right)\right] / d w\right]$ $\leqq M(1-r)^{-1+\alpha}, r<1$. Since $f[\chi(w)] d z / d w=d F[\chi(w)] / d w$, and since $|d z / d w|$ $\geqq L>0,|w| \leqq 1$, we have $M_{p}\left[f\left[\chi\left(r e^{i \theta}\right)\right]\right] \leqq M(1-r)^{-1+\alpha}, r<1$. If $k=-2$, then $F(z)$ belongs to $H(-1, \alpha, p)$ on $\gamma$; by the above argument $F[\chi(w)]$ belongs to $H(-1, \alpha, p)$ on $\gamma$; by (a), $d F\left[\chi\left(r e^{i \theta}\right)\right] / d w$ belongs to $H(-2, \alpha, p)$ on $;$; and by the argument used above, $f[\chi(w)]$ belongs to $H(-2, \alpha, p)$ on $\gamma$. If $k$ is any negative integer, repetition of the above argument gives the desired result.

In defining the class $Z(k, p)$ on $\gamma$ when $k$ is any integer we first define the class $Z(-2, p)$ as the class of functions $f(z)$ for which $M_{p}\left[f\left(r e^{i \theta}\right)\right] \leqq M(1-r)^{-1}$, $r<1$. We then define the class $Z(k, p), k \neq-2$, by using the class $Z(-2, p)$ as fundamental; the class $Z(q-2, p), q>0$, is the class of $q$ th iterated integrals of functions of class $Z(-2, p)$; the class $Z(q-2, p), q<0$, is the class of $q$ th derivatives of functions of class $Z(-2, p)$. The new definition has a natural basis in the results (b) and (c) above and in the following theorem, see Zygmund [1, Theorem 13]: A necessary and sufficient condition that a function $f(z)$ analytic interior to $\gamma$ of be class $Z(0, p)$ on $\gamma$ is that $M_{p}\left[f^{\prime \prime}\left(r e^{i \theta}\right)\right]$ $\leqq M(1-r)^{-1}, r<1$. Thus for each $p$ we have on both $\gamma$ and $C$ a sequence of classes $\cdots, Z(-3, p), Z(-2, p), Z(-1, p), Z(0, p), Z(1, p), \cdots$; the derivative and integral of a function of any class belong respectively to the next lower and next higher class. The class $Z(q-2, p), q \geqq 2$, is identical with the class $Z(k, p)$ previously defined for $k=q-2 \geqq 0$. The class $Z(k, p)$ is invariant under conformal mapping of the interior of $\gamma$ onto itself. The proof is similar to the proof given above for the invariance of the class $H(k, \alpha, p)$ under conformal mapping.

If $C$ consists of a finite number of mutually exterior analytic Jordan curves, a function $f(z)$ belongs to the class $H(k, \alpha, p)$ or $Z(k, p)$ on $C$ if $f(z)$ belongs to the respective class $H(k, \alpha, p)$ or $Z(k, p)$ on each component of $C$. 
The new classification $H(k, \alpha, p)$ and $Z(k, p)$ for all integers $k$ is especially suited to the study of Problem $\beta$, for it enables us to consider the relation between degree of approximation on $C$ and integral asymptotic conditions on $C_{\rho}$ as well as the relation between degree of approximation on $C$ and integrated Lipschitz and Zygmund conditions on $C_{\rho}$.

Theorem 3. Let $C$ and $C_{\rho}$ each consist of a finite number of mutually exterior analytic Jordan curves. If for any integer $k$ the function $f(z)$ belongs to $H(k, \alpha, p)[0<\alpha<1]$ or to $Z(k, p)[\alpha=1]$ on $C_{\rho}$, polynomials $\pi_{n}(z)$ in $z$ of $d e-$ gree $n$ exist such that

$$
\left|f(z)-\pi_{n}(z)\right| \leqq \frac{L}{n^{k+\alpha} \rho^{n}},
$$
z on $\bar{C}$.

For $k \geqq 0$, by Theorem 1 , polynomials $P_{n}(z)$ exist such that

$$
\int_{C_{\rho}}\left|f(z)-P_{n}(z)\right| p|d z| \leqq \frac{M}{n^{(k+\alpha) p}} .
$$

Let $\pi_{n}(z)$ be a polynomial in $z$ of degree $n$ which interpolates to $f(z)$ in $n+1$ equally distributed points $z_{i}$ on $C$. Let $\omega_{n}(z)=\left(z-z_{1}\right)\left(z-z_{2}\right) \cdots\left(z-z_{n+1}\right)$. Then for $z$ on $C$, by the use of theorems of Walsh and Sewell [1, Theorem 3.1 and 4.7], Hölder's inequality, and inequality (16) we have

$$
\begin{aligned}
\left|f(z)-\pi_{n}(z)\right| & \leqq \frac{1}{2 \pi} \int_{C_{\rho}} \frac{\left|\omega_{n}(z)\right|\left|f(t)-P_{n}(t)\right|}{\left|\omega_{n}(t)\right||t-z|}|d t| \\
& \leqq \frac{L_{1}}{\rho^{n}}\left\{\int_{C_{\rho}}\left|f(t)-P_{n}(t)\right| p|d t|\right\}^{1 / p} \leqq \frac{L}{n^{k+\alpha} \rho^{n}} .
\end{aligned}
$$

For $k<0$, let $F_{j}(z)$ denote the (any) $k$ th indefinite integral of $f(z)$ in the closed interior of the component $C_{\rho}^{(j)}$ of $C_{\rho}$. Then $F_{j}(z)$ belongs to the class $H(0, \alpha, p)$ or $Z(0, p)$ on $C_{\rho}^{(j)}$, and $F(z)$ defined as $F_{j}(z)$ on $C_{\rho}^{(j)}$ belongs to the class $H(0, \alpha, p)$ or $Z(0, p)$ on $C_{\rho}$. Let $p_{n}(z)$ be a polynomial of degree $n$ which interpolates to $F(z)$ in $n+1$ equally distributed points on $C$. By Theorem 1, polynomials $P_{n}(z)$ of degree $n$ in $z$ exist such that

$$
\int_{C_{\rho}}\left|F(z)-P_{n}(z)\right| p|d z| \leqq \frac{M_{1}}{n^{\alpha p}} .
$$

For $z$ on $C$, we have

$$
F(z)-p_{n}(z)=\frac{1}{2 \pi i} \int_{c_{\rho}} \frac{\omega_{n}(z)\left[F(t)-P_{n}(t)\right]}{\omega_{n}(t)[t-z]} d t .
$$

Differentiating $-k$ times with respect to $z$, we use a method of proof of Walsh and Sewell [2, Theorem 7.9], Hölder's inequality, and inequality (17) to obtain 


$$
\begin{aligned}
\left|f(z)-p_{n}^{(k)}(z)\right| & \leqq \frac{M_{2} n^{-k}}{\rho^{n}}\left\{\int_{C_{\rho}}\left|F(t)-P_{n}(t)\right| p|d t|\right\}^{1 / p} \\
& \leqq \frac{L}{\rho^{n} n^{k+\alpha}},
\end{aligned}
$$

Theorem 3 was proved by Walsh and Sewell [2, Theorem 10.5] in the special case $0<\alpha<1, p=2$, and $C_{\rho}$ the unit circle.

In the direction of a converse to Theorem 3 we state the following theorem which has been established by Walsh and Sewell [2, p. 249]; Sewell [1, Theorem 7.2]:

Theorem $3^{*}$. Let $C$ be an analytic Jordan curve. If $f(z)$ is defined on $C$, if polynomials $\pi_{n}(z)$ in $z$ of degree $n$ exist such that

$$
\int_{C}\left|f(z)-\pi_{n}(z)\right| p|d z| \leqq \frac{M}{n^{(k+\alpha+1) p} \rho^{n p}}, \quad 0<\alpha \leqq 1, \rho>1,
$$

then $f(z)$ if suitably defined is analytic interior to $C_{\rho}$ and of class $H(k, \alpha, \infty)$ on $C_{\rho}$ if $0<\alpha<1$, and of class $Z(k, \infty)$ if $\alpha=1$. If $k \geqq 0$, the inequality

$$
\left|f(z)-\pi_{n}(z)\right| \leqq \frac{M_{1}}{n^{k+\alpha}}
$$

is valid for $z$ on $\bar{C}_{\rho}$.

We give an illustration to show that the classes $H(k, \alpha, p)$ may be more useful in application to Problem $\beta$ than the older classes $H(k, \alpha, \infty)$. The function $f(z) \equiv 1 /(\rho-z)$ is of class $H(-1,1 / 2,2)$ on $\gamma_{\rho}:|z|=\rho$, for we have $\left(z=r e^{i \theta}\right)$ with $r<\rho$

$$
\int_{|z|=r}\left|f\left(r e^{i \theta}\right)\right|^{2}|d z|=\int_{0}^{2 \pi} \frac{r d \theta}{\rho^{2}-2 r \rho \cos \theta+r^{2}}=\frac{2 \pi r}{\rho^{2}-r^{2}} ;
$$

this last equality follows by identification of the integral with Poisson's integral. By Theorem 3 there exist polynomials $\pi_{n}(z)$ of respective degrees $n$ satisfying

$$
\left|f(z)-\pi_{n}(z)\right| \leqq \frac{L}{n^{-1 / 2} \rho^{n}},
$$
$z$ on $\gamma$.

This is a stronger inequality than

$$
\left|f(z)-\pi_{n}(z)\right| \leqq \frac{L}{n^{-1} \rho^{n}},
$$

which is obtained by using merely $\left|f\left(r e^{i \theta}\right)\right| \leqq 1 /(\rho-r), r<\rho$.

Bounded analytic functions. The technique we have used thus far applies with suitable modifications in the study of approximation by bounded ana- 
lytic functions; compare Walsh [2].

TheOREM 4. Let $C$ be an analytic Jordan curve which together with its interior lies in a region $D$. Let the sequence of functions $f_{n}(z)$ be analytic in $D$ and satisfy the inequalities $(k \geqq 0)$

$$
\begin{aligned}
\left|f_{n}(z)\right| & \leqq L_{1} R^{n}, z \text { in } D, & R>1, \\
\int_{C}\left|F(z)-f_{n}(z)\right|^{p}|d z| & \leqq \frac{L_{2}}{n^{(k+\alpha) p}}, & 0<\alpha \leqq 1 .
\end{aligned}
$$

Then $F(z)$ is equivalent on $C$ to a function $f(z)$ of class $H_{(-\alpha}(k, \alpha)$ if $0<\alpha<1$, and of class $Z(k, p)$ if $\alpha=1$. If $\alpha p>1$, we have

$$
\left|f(z)-f_{n}(z)\right| \leqq \frac{L_{3}}{n^{k+\alpha-1 / p}}, \quad \text { zon } \bar{C} .
$$

To prove Theorem 4, we use the method of Theorem 1 to determine polynomials $P_{q n}(z)$ of respective degrees $q n$ which approximate to $f_{n}(z)$ with degree of approximation $r^{n}, r<1$, on $C$, and define through $P_{q_{n}}(z)$ polynomials $\pi_{n}(z)$ such that

$$
\int_{C}\left|f(z)-\pi_{n}(z)\right|^{p}|d z| \leqq \frac{M}{n^{(k+\alpha) p}} ;
$$

Theorem 2 applies to complete the proof.

It is worthy of note that Theorem 2 is a consequence of Theorem 4, for if $f_{n}(z)$ is a polynomial of degree $n$ in $z$, inequality (18) is a consequence of (19) by Lemma 1.

Best approximation. For polynomials and bounded analytic functions of best approximation in the sense of least $p$ th powers we state Theorems 5 and 6 .

THEOREM 5. Let $C$ be an analytic Jordan curve and let $f(z)$ belong to $H(k, \alpha, p)$ $[0<\alpha<1]$ or $Z(k, p)[\alpha=1]$ on $C_{\rho}$ for any integer $k$. If $q_{n}(z)$ is the polynomial in $z$ of degree $n$ such that $\mu_{n}^{p}=\int_{C}\left|f(z)-q_{n}(z)\right| p|d z|$ is least, then we have

$$
\int_{C}\left|f(z)-q_{n}(z)\right| p|d z| \leqq \frac{M}{n^{(k+\alpha) p} \rho^{n p}} ;
$$$$
\left|f(z)-q_{n}(z)\right| \leqq \frac{M}{n^{k+\alpha-1 / p} \rho^{n}}, z \text { on } \bar{C}
$$$$
\left|f(z)-q_{n}(z)\right| \leqq \frac{M R^{n}}{n^{k+\alpha} \rho^{n}}, \text { z on } C_{R}, \quad 1<R<\rho ;
$$

$$
\left|f(z)-q_{n}(z)\right| \leqq \frac{M}{n^{k+\alpha-1}}, z \text { on } C_{\rho}, \quad k \geqq 1 \text {. }
$$


Inequality (a) follows from Theorem 3 and the definition of $q_{n}(z)$; inequality (b) from Theorem 3 and a theorem of Jackson [2, p. 856]; inequalities (c) and (d) follow from (a) and Theorem $3 *$.

Theorem 6. If $C$ and $D$ (bounded) are defined as in Theorem 4 , if $f(z)$ is of class $H_{p}$ on $C$, if for each $M>0$ the function $F_{M}(z)$ is the (or a) function analytic and of modulus not greater than $M$ in $D$ of best approximation to $f(z)$ on $C$ in the sense that $\mu_{M}^{p}=\int_{C}\left|f(z)-F_{M}(z)\right|^{p}|d z|$ is least, then a necessary and sufficient condition that $f(z)$ belong to $H(k, \alpha, p)[0<\alpha<1]$ or to $Z(k, p)[\alpha=1]$ on $C$ is that $\mu_{M}^{1 /(k+\alpha)} \log M$ be bounded as $M$ becomes infinite.

The sufficiency of the condition follows from Theorem 4 . The necessity of the condition follows from Theorem 1 , for if the $f_{n}(z)$ are polynomials of respective degrees $n,(18)$ is a consequence of (19) by virtue of Lemma 1, in an arbitrary bounded region $D$ containing $C$.

Extensions. The direct theorems which we have proved under the assumption that $f(z)$ belongs to $H(k, \alpha, p), k \geqq 0$, are likewise true if $\alpha$ is replaced by unity, that is, if $f^{(k)}(z)$ satisfies an integrated Lipschitz condition with $\alpha=1$; theorems in the converse direction are not valid if $\alpha=1$.

Our theorems may also be extended to include approximation by polynomials of degree $n$ in $z$ and $1 / z$ where the function approximated need not be analytic throughout the interior of $C$. We conclude by stating two results involving such polynomials, analogous to theorems proved by Walsh [5, Theorem 1] and Walsh and Elliott [2, Theorem 1].

TheOREM 7. If $C$ is an analytic Jordan curve containing the origin in its interior, a necessary and sufficient condition that $f^{(k)}(z)$ exist and satisfy an integrated Lipschitz $(0<\alpha<1)$ or Zygmund $(\alpha=1)$ condition of exponent $p$ on $C$ is that polynomials $P_{n}(z, 1 / z)$ of degree $n$ in $z$ and $1 / z$ exist such that

$$
\int_{C}\left|f(z)-P_{n}(z, 1 / z)\right| p|d z| \leqq \frac{M}{n^{(k+\alpha) p}}, \quad 0<\alpha \leqq 1 .
$$

TheOREM 8. If $C$ is an analytic Jordan curve which lies in a region $D$ and if functions $f_{n}(z)$ satisfy the conditions of Theorem 4 , then $f^{(k)}(z)$ exists on $C$ and satisfies there an integrated Lipschitz $(0<\alpha<1)$ or Zygmund $(\alpha=1)$ condition of exponent $p$.

The converse of Theorem 8 is contained in Theorem 7 provided there exist points exterior to the given region $D$ both interior and exterior to $C$; compare Lemma 1.

\section{BiBLIOGRAPHY}

John CurTiss

1. A note on the degree of polynomial approximation, Bull. Amer. Math. Soc. vol. 42 (1936) pp. 873-878. 
H. M. Elliott

1. On approximation to functions satisfying a generalized continuity condition, Trans. Amer. Math. Soc. vol. 71 (1951) pp. 1-23.

G. H. HARDY AND J. E. LitTlewood

1. Some properties of fractional integrals. I., Math. Z. vol. 27 (1928) pp. 565-608.

2. A convergence criterion for Fourier series, Math. Z. vol. 28 (1928) pp. 612-634.

3. Some properties of fractional integrals. II., Math. Z. vol. 34 (1932) pp. 403-439.

D. JACKSON

1. On the degree of convergence of Sturm-Liouville series, Trans. Amer. Math. Soc. vol. 15 (1914) pp. 439-466.

2. On certain problems of approximation in the complex domain, Bull. Amer. Math. Soc.

E. S. QUADE vol. 36 (1930) pp. 851-857.

1. Trigonometric approximation in the mean, Duke Math. J. vol. 3 (1937) pp. 529-543.

F. RIESZ

1. Über die Randwerte einer analytischen Funktion, Math. Z. vol. 18 (1923) pp. 87-95.

WALter RUdiN

1. Analytic functions of class $H_{p}$, Trans. Amer. Math. Soc. vol. 78 (1955) pp. 46-66.

W. E. SEWELL

1. Degree of approximation by polynomials in the complex domain, Annals of Mathematics Studies, no. 9, Princeton, 1942.

V. SmirnofF

1. Sur les formules de Cauchy et de Green et quelques problèmes qui s'y rattachent, Bull. Acad. Sci. URSS. Sér. Math. vol. 7 (1932) pp. 337-371.

C. J. de la Vallée Poussin

1. Leçons sur l'approximation des fonctions d'une variable réelle, Paris, 1919.

J. L. WALSH

1. Interpolation and approximation by rational functions in the complex domain, Amer. Math. Soc. Colloquium Publications, vol. 20, 1955.

2. Note on approximation by bounded analytic functions, Proc. Nat. Acad. Sci. U.S.A. vol. 37 (1951) pp. 821-826.

3. Degree of approximation to functions on a Jordan curve, Trans. Amer. Math. Soc. vol. 73 (1952) pp. 447-458.

J. L. Walsh and H. M. Elliott

1. Polynomial approximation to harmonic and analytic functions: generalized continuity conditions, Trans. Amer. Math. Soc. vol. 68 (1950) pp. 183-203.

2. Degree of approximation on a Jordan curve, Proc. Nat. Acad. Sci. U.S.A. vol. 38 (1952) pp. 1058-1066.

J. L. Walsh and W. E. Sewell

1. Sufficient conditions for various degrees of approximation by polynomials, Duke Math. J. vol. 6 (1940) pp. 658-705.

2. On the degree of polynomial approximation to analytic functions: Problem $\beta$, Trans. Amer. Math. Soc. vol. 49 (1941) pp. 229-257.

D. W. WESTERN

1. Inequalities of the Markoff and Bernstein type for integral norms, Duke Math. J. vol. 15 (1948) pp. 839-869.

\section{A. ZYGMUND}

1. Smooth functions, Duke Math. J. vol. 12 (1945) pp. 47-76.

HaRVARd UnIVERSITy,

Cambridge, Mass.

Wellesley College,

Wellesley, Mass. 